EPJ Web of Conferences 32, 01006 (2012)

DOI: $10.1051 /$ epjconf/20123201006

(C) Owned by the authors, published by EDP Sciences, 2012

\title{
Capabilities of the ITER Electron Cyclotron Equatorial Launcher for Heating and Current Drive
}

\author{
D. Farina ${ }^{1, \text { a }}$, L Figini ${ }^{1}$, M Henderson ${ }^{2}$, G. Ramponi ${ }^{1}$, and G Saibene ${ }^{3}$ \\ 1 Istituto di Fisica del Plasma, CNR, EURATOM-ENEA-CNR Association, 20125 Milano, Italy \\ 2 ITER Organization, 13108 Saint-Paul-lez-Durance, France \\ 3 Fusion for Energy, 08019 Barcelona, Spain
}

\begin{abstract}
The ITER Electron Cyclotron Equatorial Launcher is designed to be one of the heating systems to assist and sustain the development of various ITER plasma scenarios starting with the very first plasma operation. Here the capabilities for Heating and Current Drive of this system are reviewed. In particular, the optimum launching conditions are investigated for two scenarios at burn, comparing toroidal and poloidal steering options. Then, the EC capabilities are investigated for different plasma parameters corresponding to various phases of the ITER plasma discharge, from current ramp-up up to burn, and for a wide range of magnetic field, focusing in particular on the EC potential for heating and for $\mathrm{L}$ to $\mathrm{H}$-mode assist. It is found that the EC system can contribute to a wide range of heating scenarios during the ramp-up of the magnetic field, significantly increasing the applicable range as a function of magnetic field.
\end{abstract}

\section{Introduction}

Electron Cyclotron (EC) Heating and Current Drive (H\&CD), at $170 \mathrm{GHz}, 20 \mathrm{MW}$, is one of the heating systems foreseen to assist and sustain the development of various ITER plasma scenarios starting with the very first plasma generated in ITER. This will be accomplished by launching the EC power via the Equatorial Launcher (EL), with the primary goal of heating and $q$ profile tailoring in the central plasma region. The EL setup is made up of three mirror assemblies (TOP, MID, BOTTOM), with a steering mirror at the end of each assembly, located at the same major radial position $R$ and three different $z$, with eight beams for each mirror [1]. In the present design the toroidal injection angle $\beta$ is varied in the range $20^{\circ} \leq|\beta| \leq 45^{\circ}$ while the poloidal angle $\alpha$ is kept fixed with the beams from MID mirror being injected horizontally $\left(\alpha=0^{\circ}\right)$ and the beams from TOP and BOTTOM mirror poloidally tilted towards plasma center by $\alpha= \pm 5^{\circ}$. Beams from the MID mirror are injected in the opposite toroidal direction with respect to the other mirrors to provide counter current drive.

The EL capabilities have been investigated in recent years focusing on full-field scenarios at burn [2-4]. The analysis performed at full field in the burn phase has pointed out that the EL is capable to drive a current up to mid radius, depending on the plasma density and temperature, but not at larger radii. Here, first results are presented of a dedicated beam tracing analysis aimed at determining the optimum launch conditions for maximizing the driven current from the (EL) and improve the power deposition accessibility to beyond mid radius. This goal can be accomplished by steering poloidally the EC beams, aiming at outer surfaces.

In addition, we present ECRH\&CD results obtained for various plasma parameters corresponding to different phases of the ITER plasma discharge, from current ramp-up up to burn, and for a wide range of magnetic field, focusing in particular on the EC potential for heating $(\rho<0.5)$ and for $\mathrm{L}$ to H-mode assist $(\rho<0.8)$.

\footnotetext{
a e-mail: farina@ifp.cnr.it
} 

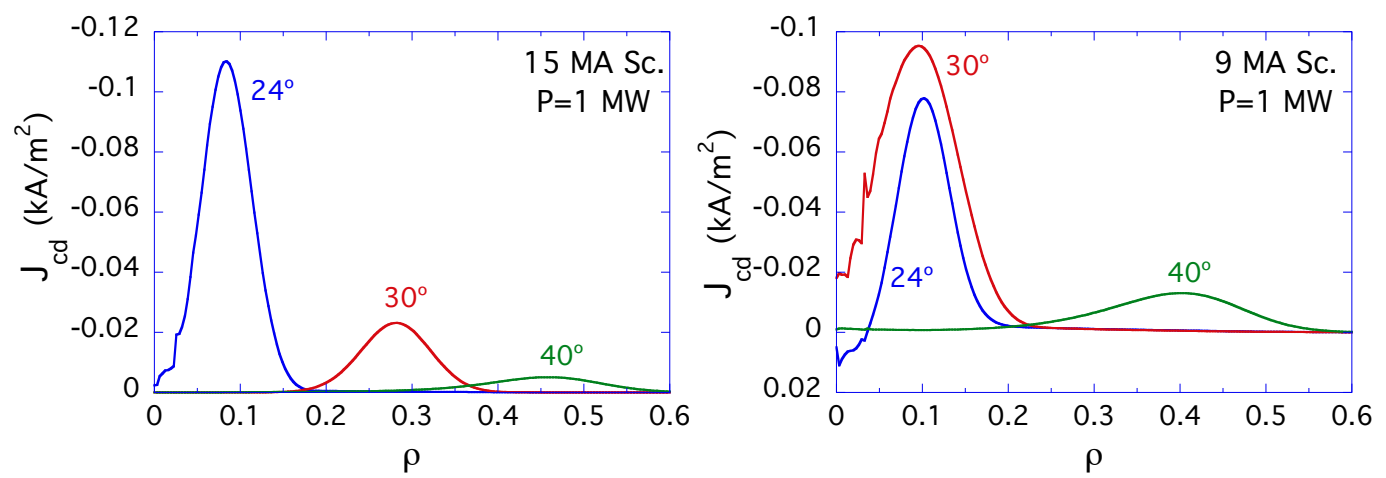

Fig. 1. EC current density profiles from the MID row for toroidal angles $24^{\circ}, 30^{\circ}$ and $40^{\circ}$, and $1 \mathrm{MW}$ of injected power and the $15 \mathrm{MA}$ scenario (left) and 9 MA scenario (right). Note the different localization in the two scenarios.

Simulations are performed by means of beam tracing code GRAY [5], using either a reference beam [7] or single ray, since for large enough toroidal angles Doppler shift effects are dominant. Driven current and absorbed power density profiles, $J_{c d}(\rho)=d I_{c d} / d A, p(\rho)=d P / d V$, are characterized in term of suitable averaged radii

$$
\langle\rho\rangle_{p}=\frac{\int d V \rho p(\rho)}{\int d V p(\rho)}, \quad\langle\rho\rangle_{j}=\frac{\int d A \rho J_{c d}(\rho)}{\int d A J_{c d}(\rho)}
$$

and the profile widths are defined in terms of the variance. These definitions apply to the case of non monotonic profiles, and the average values coincide with the peak values in case of almost Gaussian profiles.

\section{ECCD in ITER scenarios at burn}

Investigation of the ECCD capabilities has been performed by considering injection of EC beams polarized as ordinary mode into two ITER Scenarios, a 15 MA ELMy H-mode and a 9 MA Steady State scenarios at burn [7]. The two scenarios are characterized by different magnetic equilibrium and plasma profiles, the $9 \mathrm{MA}$ Scenario has a larger electron temperature and a lower density $\left(T_{e 0}=34\right.$ $\mathrm{keV}, n_{e 0}=7 \times 10^{19} \mathrm{~m}^{-3}$ ) with respect to $15 \mathrm{MA} \mathrm{H}$-mode scenario $T_{e 0}=27 \mathrm{keV}, n_{e 0}=1 \times 10^{20} \mathrm{~m}^{-3}$ ), a slightly inverted $q$ profile, and the equilibrium is shifted outward.

Similar scenarios have been already investigated in the previous papers [2,3], and here we present the results obtained with the more accurate evaluation of ECCD performed by taking into account momentum conservation [6]. Larger ECCD values by approximately $15 \%$ of CD efficiency are found in the central region of both scenarios. The EC driven current is as high as $\approx 30 \mathrm{kA} / \mathrm{MW}$ for the 15 MA scenario and up to $\approx 60 \mathrm{kA} / \mathrm{MW}$ for the $9 \mathrm{MA}$ scenario. The maximum current drive from the MID row is obtained around $\rho=0.1$ for $\beta \approx 25^{\circ}$ in the $15 \mathrm{MA}$ scenario while at $\rho=0.25$ at a larger toroidal angle $\beta \approx 33^{\circ}$ for the 9 MA scenario. The high ECCD obtained in this latter case is due to the larger $T_{e} / n_{e}$ ratio (by a factor 2), i.e. to the larger CD efficiency. However, due to the larger $T_{e}$ the 9 MA case suffers from high second harmonic parasitic absorption in the low field side, that reduces the ECCD at lower $\beta$ and $\rho$, and may even result in double peaked $J_{c d}$ profiles. Examples of driven current density profiles are shown in Figure 1. It is important to note that ECRH\&CD is effective only in the central plasma region, i.e., up to $\rho \approx 0.45$ for both scenarios, as it is apparent from the plots.

To analyze the full EC capabilities by injecting from the equatorial launcher, ECCD from a full scan of poloidal $\alpha$ and toroidal $\beta$ injection angles has been studied for both scenarios and all the three launching mirrors, with the goal to verify whether modifying the beam steering angles from a pure toroidal sweep to either a poloidal or combined poloidal-toroidal steering would improve the ECCD performance especially at mid radius. The results of these simulations are reported in Figure 2 for 

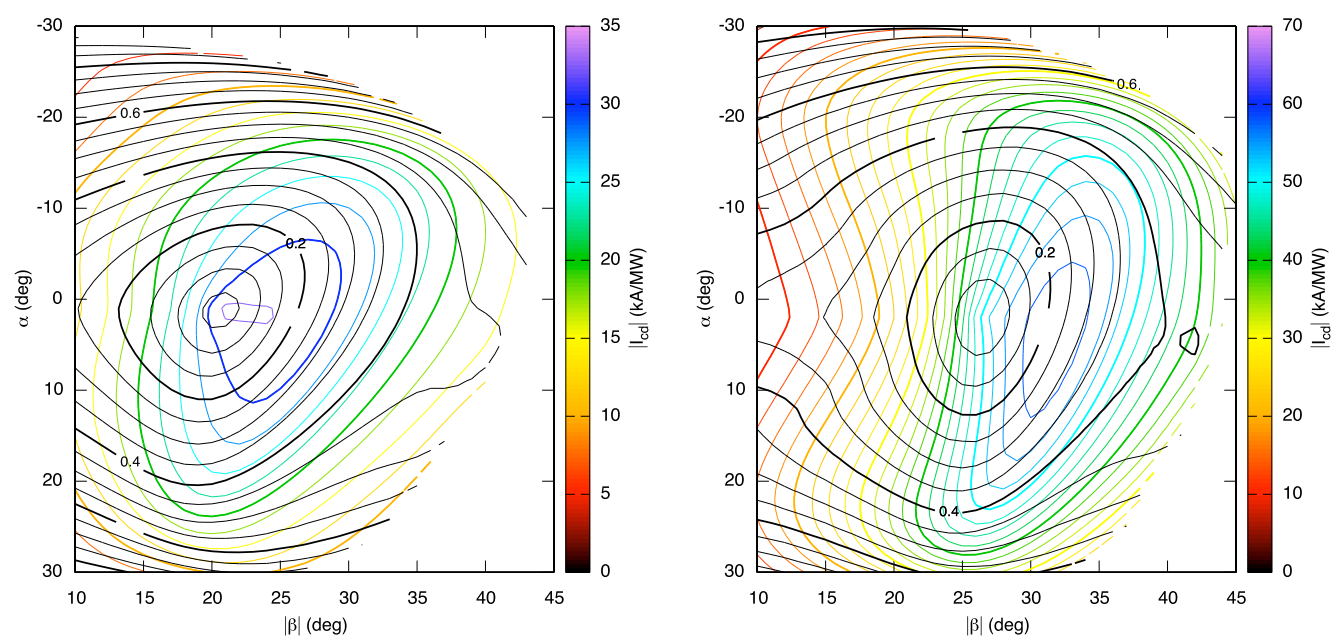

Fig. 2. Contours of the radial ECCD location (black solid lines) and of the driven current (coloured lines) as a function of the poloidal $\alpha$ and toroidal $\beta$ injection angles, for the $15 \mathrm{MA}$ (left) and $9 \mathrm{MA}$ (right) scenario.

injection from the EL mid row, where the contours of the radial ECCD location and the contours of the driven current are reported. Similar $I_{c d}$ results are obtained from the other rows at poloidal angles shifted upward or downward with respect to this case. The present design with pure toroidal steering corresponds to a "cut" at constant $\alpha$. As it may be appreciated from the plots, various ECCD efficiency at different locations can be obtained varying the path in the $(\alpha, \beta)$ plane.

In Figure 3 the same results are plotted in the plane $\left(\rho, I_{c d}\right)$ and compared with the current driven from the Upper Launcher (UL), that is a second ITER EC system devoted to MHD control in the outer plasma region. The dotted plots are the results from pure toroidal steering.
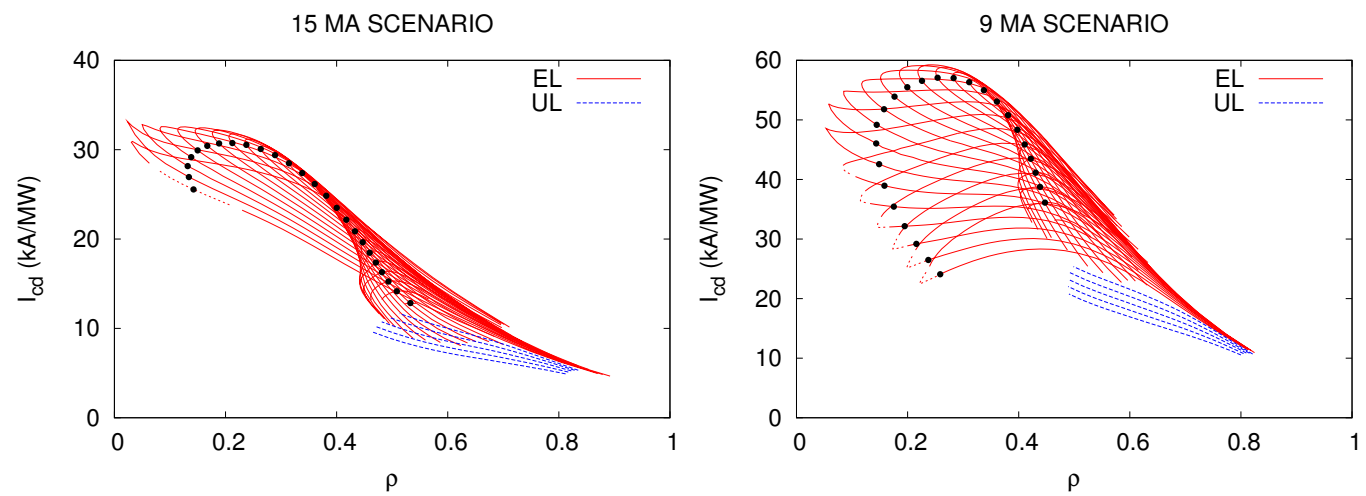

Fig. 3. Driven current per unit injected power versus $\rho$ from the EL (red solid lines) for $20^{\circ} \leq \beta \leq 45^{\circ}$ and from the UL (blue dotted lines) for $18^{\circ} \leq \beta \leq 22^{\circ}$. The black dots correspond to the present EL design.

It is clearly seen that toroidal steering provides almost the maximum current in the central plasma region up to $\rho \approx 0.4$, then the efficiency drops sharply and poloidal steering would be required to go beyond mid radius. In the outer region the EL is still more efficient than the UL as far as the total driven current is concerned, mainly due to the higher $\beta$ value used. The results show that poloidal steering would allow to extend the potential of the EC system at full field at burn. A major constraint to the choice of the toroidal angle $\beta$ is given by an additional functional capability required to the Equatorial Launcher, that is EL is to deliver EC power in the early stage of an ITER discharge to assist plasma breakdown and startup. To perform this goal it is necessary that the EC beams are injected as OM 
and undergo reflection and mode conversion to XM at the inner wall, to be efficiently absorbed. This process requires oblique injection of $\mathrm{EC}$ beams with a maximum toroidal angle $\approx 23^{\circ}$. It is found that pure poloidal steering at $\beta \simeq 20^{\circ}$ would provide a much larger radial range for ECCD but a much lower driven current in case of the 9 MA scenario close to plasma center, because of the second harmonic parasitic absorption.

\section{ECRH\&CD in an ITER inductive scenario up to start of burn}

To study the EC potential at various stages of an ITER discharge and in a wide range of magnetic field, injection of EC beams polarized both as ordinary mode (OM) and as extraordinary mode (XM) is considered in an inductive scenario at $15 \mathrm{MA}$ at different time slices (see Table 1), both in $\mathrm{L}$ and in $\mathrm{H}$-mode, for which quite different EC interaction conditions are expected. The early time slices of the ITER discharge (up to $80 \mathrm{~s}$ ) are characterized by a much lower temperature and density and slightly different equilibrium with respect to the 15 MA scenario at burning investigated in Section 2.

Table 1. Plasma parameters for a 15 MA scenario at various time slices

\begin{tabular}{lllll}
\hline Case & $t(\mathrm{~s})$ & $n_{e 0}\left(10^{19} \mathrm{~m}^{-3}\right)$ & $T_{e 0}(\mathrm{keV})$ & $I_{p}(\mathrm{MA})$ \\
\hline (a) & 11.7 & 3.7 & 0.75 & 4.6 \\
(b) & 58 & 11.5 & 3.00 & 8.0 \\
(c) & 80 & 15.0 & 4.00 & 14.4 \\
(d) & 130 & 15.0 & 8.50 & 29.0 \\
\hline
\end{tabular}

\subsection{ECCD at different time slices}

The EC driven current as a function of the radius $\rho$ is shown in Figure 4 for cases (a) to (d), and injection from the MID row. Only results corresponding to at least $95 \%$ of absorbed power are plotted.

At the early stage of the discharge only XM is efficiently absorbed at first pass and a quite high $I_{c d}$ is driven due to the low plasma density. XM interaction at first harmonic is usually prevented when the EC beams are injected from the low field side because of the presence of the right cutoff, that is located in the lfs with respect to the cold resonance. However, in oblique injection it may happen that $\mathrm{XM}$ is absorbed even in the presence of the XM cut-off, that is before being reflected, especially in high temperature and low density plasmas where the cutoff surface is quite close to the EC resonance surface, and upshifted absorption may occur along the trajectory far ahead of the cold resonance. When this occurs, XM turns to be more efficient than the OM because of the favorable density scaling.

During ramp-up, with increasing electron temperature and density, both OM and XM absorption may occur, although with a different spatial localization. At the end of the ramp-up phase $t=80 \mathrm{~s}$ and at a longer time, only $\mathrm{OM}$ can penetrate into the plasma close to the $\mathrm{EC}$ resonances. At the last time investigated $t=130$, EC current up to $\approx 40 \mathrm{kA} / \mathrm{MW}$ is driven in a wide radial interval $(\rho \lesssim 0.6)$ with an efficiency slightly larger than that reported in Section 2, due to the higher temperature and lower density of this scenario. Note that the CD efficiency in the central region is remarkably lower at $t=130 \mathrm{~s}$ than at $t=80 \mathrm{~s}$, due mainly to parasitic second harmonic absorption occurring in high temperature plasma, that reduces the effective power available for ECCD at first harmonic.

\subsection{EC heating potential at nominal and reduced fields}

The potential of the EC system for heating has been analyzed in a wide interval of magnetic field lower than the nominal one $\left(2 \mathrm{~T} \leqslant B_{0} \leqslant 5.3 \mathrm{~T}\right)$, for both $\mathrm{OM}$ and $\mathrm{XM}$ injection at the four time slices 

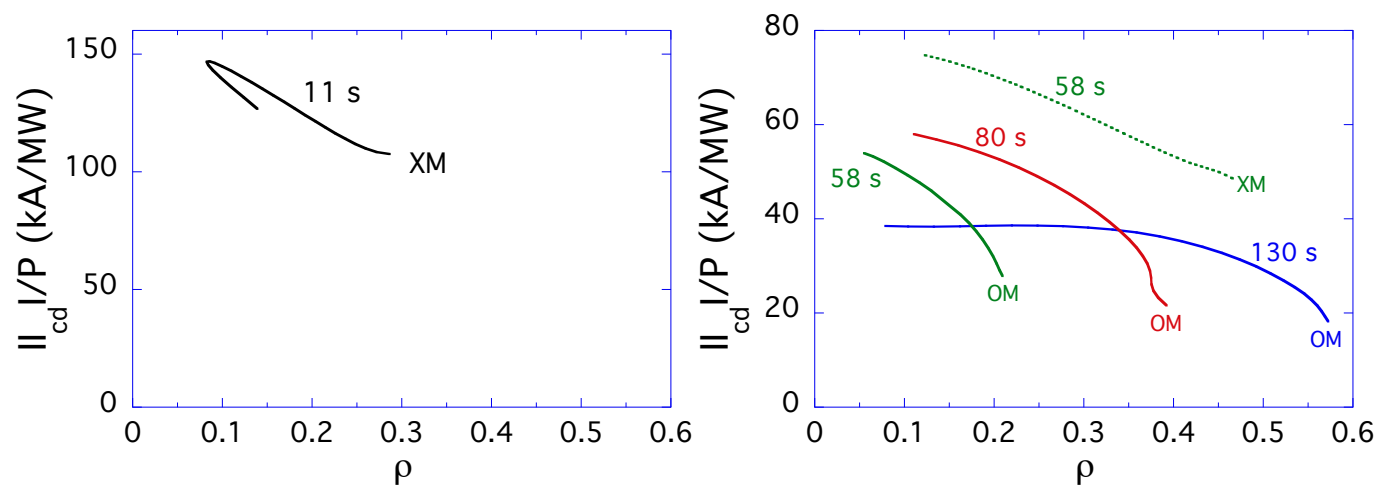

Fig. 4. Driven current as a function of $\rho$ for the four time slices of Table 1 From MID row . EC waves are injected polarized as XM at 11s (left), both as XM and $\mathrm{OM}$ at $58 \mathrm{~s}$, as $\mathrm{OM}$ at 80 sand $130 \mathrm{~s}$ (right). Only cases corresponding to more than $95 \%$ of absorbed power are shown in the plot.

of the discharge considered in Table 1. Variants of the four cases at lower toroidal magnetic fields are obtained rescaling the magnetic field, the plasma current, and the plasma pressure by the same factor, keeping the edge safety factor $q_{95}$ and the plasma beta constant, i.e., without changing the magnetic topology. Plasma density and temperature are scaled keeping $n / n_{G R W}=$ const, that implies that also $n_{e}$ and $T_{e}$ are scaled as $B_{0}$. We note that this scaling procedure is not consistent, and self-consistent simulations at lower magnetic field would be required especially for the scenario at burn, i.e., case (d). Due to lack of transport simulations at reduced field, here we limit ourselves to this simplified picture.

The behaviour of the power deposition as a function of the magnetic field is shown in Figure 5, where the average deposition radius $\langle\rho\rangle_{p}$ is plotted versus $B_{0}$ for a scan in the toroidal angle. The results can be explained in terms of the dependence of the OM and XM optical depths and of the locations of the EC resonance and of the XM cutoff in the ITER plasma. Note that at $B_{0} \approx 4 \mathrm{~T}$, the first and second harmonic resonances are located at the hfs and lfs plasma boundary, respectively.

OM power absorption is efficient at large temperature and density, and at large $B_{0}>4 \mathrm{~T}$, when interaction occurs as first harmonic (OM1), while XM power absorption is efficient at low density also at the first harmonic, as it is seen in cases (a) and (b), and at low $B_{0}<3.6 \mathrm{~T}$ (as XM2 and/or XM3) in all cases (a) to (d). Full power absorption is found in the whole magnetic range investigated regardless of the injected mode apart from case (a), i.e., at a relatively early stage of the discharge, where there are gaps in the effective magnetic field range, for $B_{0}<2.5 \mathrm{~T}$ and close to $4 \mathrm{~T}$, where absorption is localized quite close to the plasma boundary. However in these conditions, the results are sensitive to the actual details of the kinetic profiles, so that the validity is more qualitative than quantitative.

In conclusion, it is found that the EC system is applicable i) for central heating (inside mid radius) for the majority of the toroidal magnetic field range from half to full field, and ii) for assisting in $\mathrm{L}$ to $\mathrm{H}$-mode transition aside from a narrow range between $3.75 \mathrm{~T}$ and $4.0 \mathrm{~T}$.

\section{Conclusions}

The EC capabilities of the EL have been evaluated concentrating mainly on two aspects. The first refers to a possible improvement of the launching setup to allow EC interaction at larger radii from the EL, with the goal to assist L-H transition. This could be achieved by changing from toroidal to poloidal steering. It has been shown that a pure poloidal steering launcher might increase effective EC deposition beyond mid radius, at the cost of less efficiency in the plasma core at high temperature and less flexibility at reduced field operation.

The second aspect investigated is the potential at stages far from flat-top and/or at reduced magnetic field. From the overall analysis, it is found that the EC system can contribute to a wide range of heating scenarios, significantly increasing its flexibility as compared to the traditional views that the EC has limited applicability around the two narrow bands of magnetic field, corresponding to nominal field 

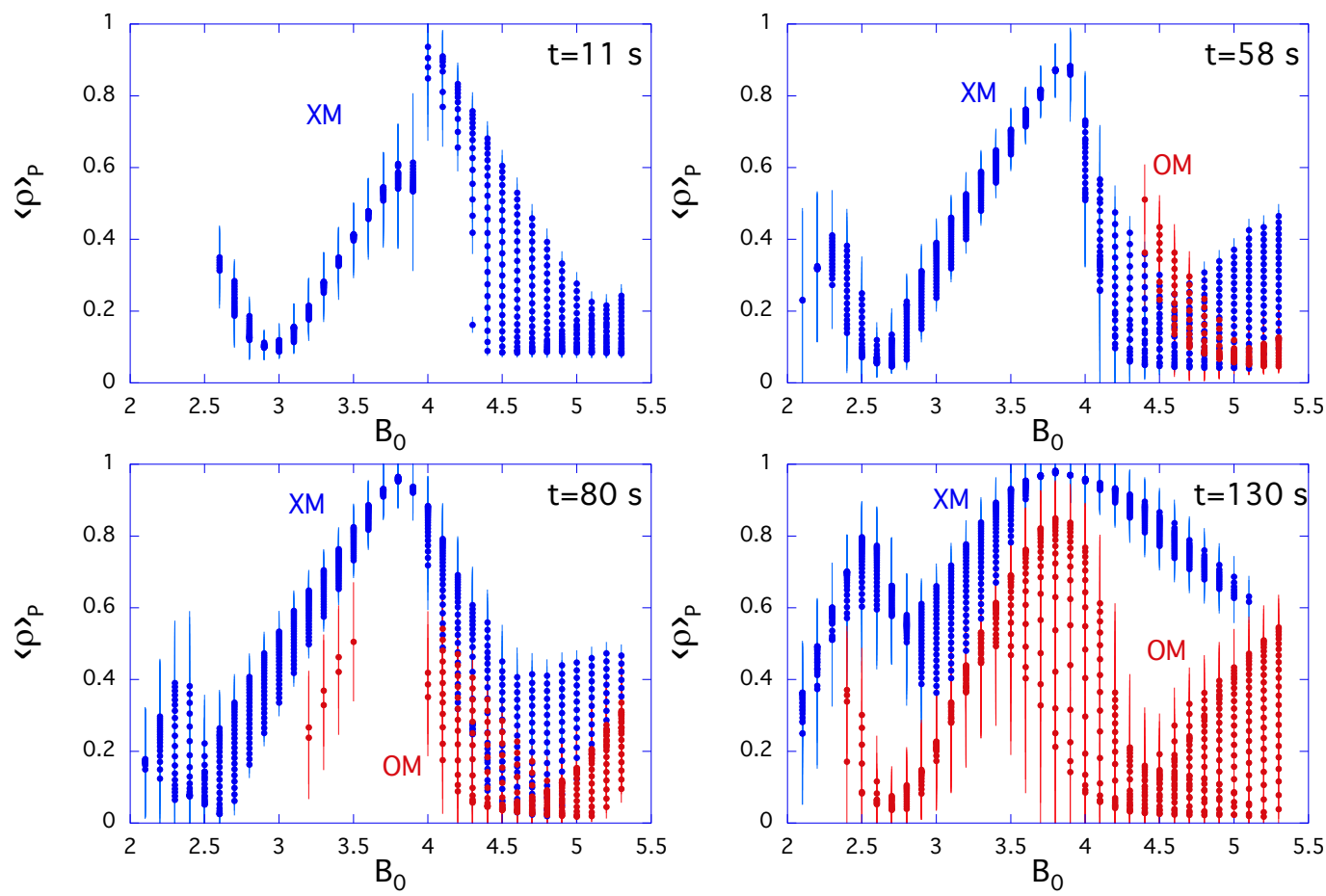

Fig. 5. Average deposition radius versus magnetic field for the four cases of Table 1 for $P_{a b s}>95 \%$. Each point corresponds to a single run with given toroidal angle $\beta$, and to OM (red) or XM (blue) polarization. The error bars indicate the profile width.

and half field. Predictions of H\&CD by EC during current ramp-up conditions at nominal magnetic field show that the EC system can achieve high to full absorption in spite of the reduced density and temperature profiles expected in these conditions, implying applicability of the EC system for control of the temperature and current profiles as well as ramp-up assist.

The authors would like to thank Alberto Loarte and Thomas Casper at ITER for useful discussions and for providing the ITER scenarios on which the analysis presented in Section 3 is based. Moreover, the authors acknowledge support of Ken Kajiwara and Koji Takahashi of JAEA for the EL beam and launcher description.

Part of this work has been carried out within the framework of ITER Service Contracts. Some of the authors are supported in part by the EURATOM-ENEA-CNR Association. The views and opinions expressed herein do not necessarily reflect those of the European Commission.

\section{References}

1. K Takahashi et al, Fusion Sci. Technol. 471 (2005)

2. G. Ramponi et al, Fusion Sci. Technol., 52, (2007) 193

3. G. Ramponi et al, Nucl. Fusion, 48, (2008) 054012

4. R. Prater R, J. Phys.: Conf. Ser. 25, (2005) 257

5. D. Farina, Fusion Sci. Technol., 52, (2007) 154

6. N. B. Marushchenko et al, Fusion Sci. Technol., 55, (2009) 180

7. D. Farina et al, Nucl. Fusion, 52, (2012) 033005 